\title{
Internet der Dienste
}

\author{
DOI 10.1007/s11576-009-0187-5
}

\section{Die Autoren}

\author{
Prof. Dr. Peter Buxmann \\ Technische Universität Darmstadt \\ Fachgebiet Information Systems \\ Hochschulstraße 1 \\ 64289 Darmstadt \\ Deutschland \\ buxmann@is.tu-darmstadt.de
}

Prof. Dr. Thomas Hess

Ludwig-MaximiliansUniversität München Institut für Wirtschaftsinformatik und Neue Medien

Ludwigstr. 28

80539 München

Deutschland

thess@bwl.Imu.de

\section{Dr. Rainer Ruggaber \\ SAP AG}

SAP Research Center CEC Karlsruhe Vincenz-Prießnitz-Str. 1

76131 Karlsruhe

Deutschland

rainer.ruggaber@sap.com

This article is also available in English via http://www.springerlink.com and http://www.bise-journal.org: Buxmann P, Hess T, Ruggaber R (2009) Internet of Services. Bus Inf Syst Eng. doi: 10.1007/s12599-009-0066-z.
Weltweit ist ein starkes Wachstum des Dienstleistungssektors zu verzeichnen. So wurden 2008 in der Bundesrepublik Deutschland etwa 70 Prozent der gesamtwirtschaftlichen Wertschöpfung mit Dienstleistungen generiert. Der Zuwachs gegenüber 2007 betrug rund 50 Milliarden Euro. Eine vom deutschen Bundeswirtschaftsministerium in Auftrag gegebene Studie kommt zu dem Ergebnis, dass Wachstumspotenzial insbesondere bei IT-Dienstleistungen existiert.

Vor diesem Hintergrund besteht die Vision des „Internets der Dienste“ darin, Anbietern die Möglichkeit zu geben, ihre Dienstleistungen über das Netz anzubieten. In Abhängigkeit vom Digitalisierungsgrad dieser Dienstleistungen können die Angebote weltweit zur Verfügung gestellt und nachgefragt werden. Das Internet der Dienste besteht dabei aus Teilnehmern, einer Infrastruktur für Dienste, Geschäftsmodellen und den Diensten selbst. Dabei werden Dienste von verschiedenen Anbietern bereitgestellt, zu Mehrwertdiensten verknüpft, an Nutzer und Konsumenten vermittelt und von diesen über verschiedene Kanäle in Anspruch genommen.

Derartige Dienste können sowohl auf der fachlichen als auch auf der infrastrukturellen Ebene angesiedelt sein. Neben technischen Aspekten existiert in diesem Umfeld auch eine Vielzahl ökonomischer Fragestellungen: Wie sehen tragfähige Geschäftsmodelle für die Anbieter derartiger Dienste aus? In welchem Umfang sollten Schnittstellen bzw. der Zugang zu Plattformen offengelegt werden? Welche Position können klassische Software- oder Medienanbieter in derartigen Märkten übernehmen? Wie lässt sich die Abrechnung effizient organisieren? Inwieweit ist eine Regulierung der Servicemärkte ökonomisch geboten? Interessant ist auch die Frage, wer derartige Dienste eigentlich anbietet. Natürlich sind „klassische“ Unternehmen, wie SAP, Oracle, Microsoft oder Google, unter den Anbietern. Daneben werden zunehmend auch Dienste bereitgestellt, hinter denen kein klassisches Unternehmen steht. Aktuell kann man das z. B. bei den Services für das iPhone oder auch für Plattformen wie Facebook beobachten.

In der Wirtschaftsinformatik existiert eine Vielzahl von Projekten, die sich dem Themenfeld des „Internets der Dienste“ widmen. Das prominenteste ist sicherlich das vom deutschen Bundesministerium für Wirtschaft und Technologie geförderte Projekt THESEUS, das sich sowohl mit technischen als auch mit ökonomischen Fragestellungen beschäftigt. Darüber hinaus gibt es an verschiedenen Standorten weitere Initiativen, die sich auf einzelne Aspekte, wie z. B. Sicherheit, konzentrieren. Unser Ziel ist es, Ihnen mit diesem Schwerpunktheft einen Überblick über diese Aktivitäten zu geben.

Der Beitrag von Benjamin Blau, Clemens van Dinther, Tobias Conte, Yongchun Xu und Christof Weinhardt von der Universität Karlsruhe (TH) und dem Forschungszentrum Informatik untersucht die Hauptkomponenten von Service Value Networks. Zur Koordination solcher Netzwerke wird von den Autoren ein skalierbarer, multidimensionaler, auktionsähnlicher Mechanismus entwickelt, welcher insbesondere die Allokation und Preissetzung komplexer Dienste organisiert.

Alexander Benlian, Thomas Hess und Peter Buxmann von der LMU München und der Technischen Universität Darmstadt thematisieren in ihrem Artikel mit Softwareas-a-Service (SaaS) ein relativ neues Paradigma der Bereitstellung von Unternehmenssoftware als Service. Im Rahmen einer großzahligen Studie untersuchen sie verschiedene Einflussfaktoren auf die Entscheidung, SaaS-Angebote einzusetzen.

Daniel Oberle, Nadeem Bhatti, Saartje Brockmans, Michael Niemann und Christian Janiesch von SAP Research CEC Karlsruhe, Fraunhofer IGD Darmstadt, ontoprise GmbH Karlsruhe, Technische Universität Darmstadt und SAP Research CEC Brisbane befassen sich mit der Strukturierung und Organisation von Informationen im „Internet der Dienste". Eingebettet ist dieser Forschungsschwerpunkt in das THESEUS/TEXO Projekt. 
In der Rubrik WI - State of the Art beschäftigen sich Christof Weinhardt, Arun Anandasivam, Benjamin Blau, Nikolay Borissov, Thomas Meinl, Wibke Michalk und Jochen Stößer von der Universität Karlsruhe (TH) mit dem Thema Cloud-Computing.

Das WI - Schlagwort von Nils-Holger Schmidt, Koray Erek, Lutz M. Kolbe und Rüdiger Zarnekow von der Georg-August-Universität Göttingen und der Technischen Universität Berlin befasst sich mit nachhaltigem Informationsmanagement.

Wir danken den Autoren unseres Schwerpunkthefts und natürlich auch allen, deren Beiträge nicht angenommen werden konnten. Ebenso gilt unser Dank den Gutachterinnen und Gutachtern, die sich teilweise in mehreren Runden differenziert mit den eingereichten Manuskripten beschäftigt haben. Weiterhin bedanken wir uns bei der Redaktion der Zeitschrift WIRTSCHAFTSINFORMATIK für die Übersetzung der Artikel. Darüber hinaus gilt unser Dank Janina Ristl von der Technischen Universität Darmstadt, die uns bei der Koordination des Schwerpunkthefts hervorragend unterstützt hat. Wir wünschen Ihnen eine interessante und inspirierende Lektüre.

Peter Buxmann

Thomas Hess Rainer Ruggaber 


\section{COMMERZBANK}

\section{Die beste Antwort auf}

stärkere Herausforderungen

sind stärkere Leistungen.

Eine neue Bank für Deutschland.

Und ein starker Partner für die Wirtschaft.

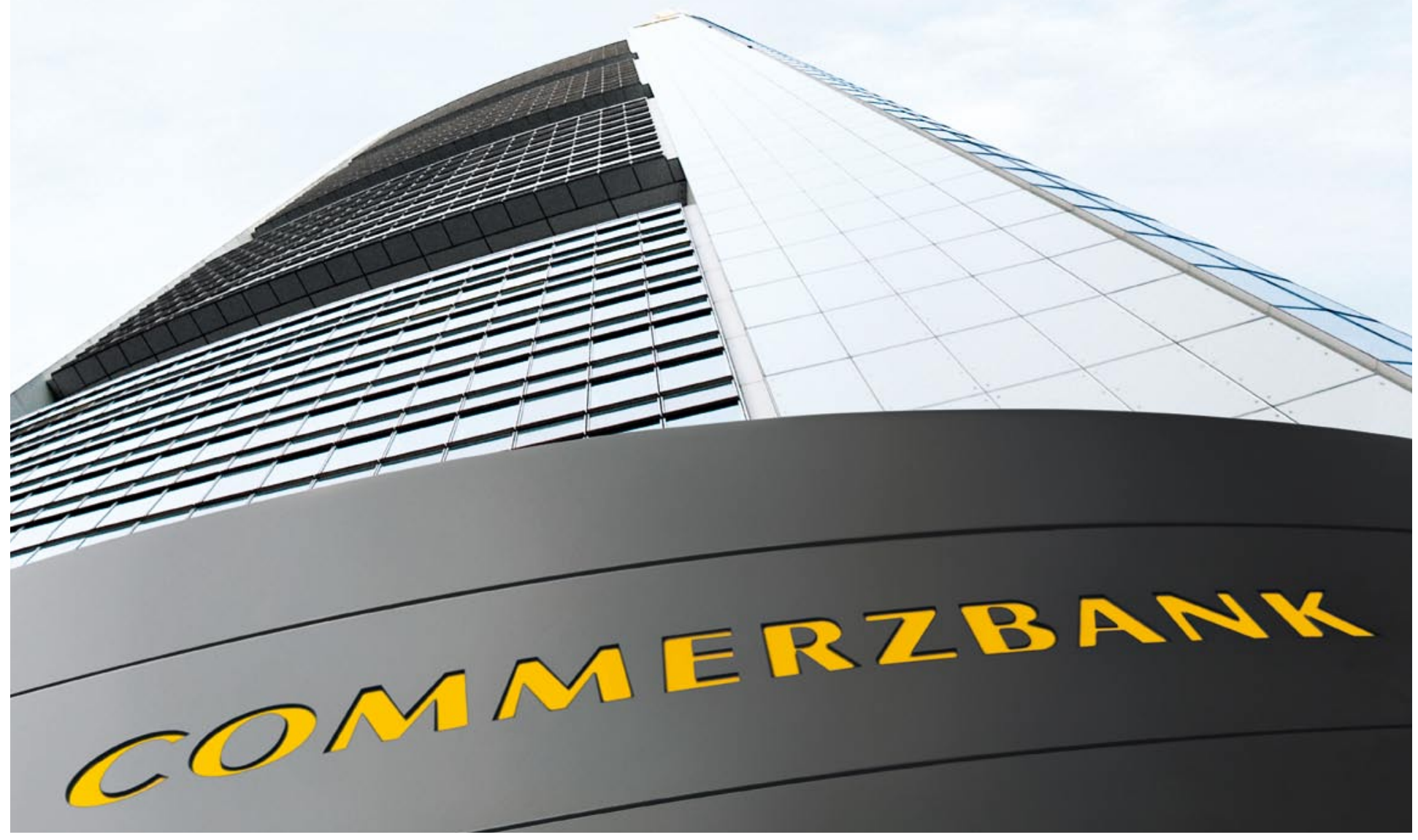

Durch den Zusammenschluss von Commerzbank und Dresdner Bank entsteht eine neue Größe im deutschen Bankenmarkt. Die neue Commerzbank wird die Wettbewerbsfähigkeit des Wirtschaftsstandorts Deutschland stärken - als die Hausbank für Privat- und Firmenkunden in Deutschland.

Gemeinsam übernehmen wir Verantwortung. Als leistungsstarke Bank und als verlässlicher Partner für die deutsche Wirtschaft. 